\title{
Functions of hereditary immunity and xenogamy in cancer origin and pandemic spread
}

\author{
Sergey N. Rumyantsev
}

Department of Evolutionary Immunology, Andent, Inc., Jersey City, USA; rumyan1@yahoo.com

Received 1 August 2011; revised 18 August 2011; accepted 31 August 2011.

\begin{abstract}
The efficacy of means exploited currently for cancer prevention and treatment appeared to be very low. New insights into the origin of the disease are sorely needed. The present article synthesizes the results from integrative reconsideration of actual data on cancer from the viewpoint of recent developments in pathology, epidemiology, immunology, genetics, and evolution. In contrast to the $\mathbf{8 0}$ years old hypothesis of somatic mutative origin of carcinogenesis, the revealed set of evidence showed the origin of cancerous clones is based on inherent constitutional incongruence between the regulators of cell physiology and their targets realized in inherent immunity of cancerous cells to normal regulation of cell replication and tissue growth. The incongruence arises out of both genome mutations which led to interethnic differences in the regulator-receptor structures and intercourse between ethnoses, the regulator-receptor evolution of which has been processed to deal with different ecologic conditions. The current pandemic spread of cancer is brought about growing expansion of interethnic xenogamy favored by growing industrialization, urbanization, globalization, and migration. The proposed hypothesis of genome intrusion in the origin of cancer induces new research ideas and proposals for cancer prevention and therapy.
\end{abstract}

Keywords: Biodiversity; Cancerous Genealogy; Carcinogenesis; Genomic Mutations; GI-hypothesis; Heterozygosity; Regulator-Receptor System; Self-Reproduction; Somatic Mutations

\section{INTRODUCTION}

Although the rare appearance of cancer disease could happen far long before the descent of human, its written history starts from the very beginning with Egyptian papyrus of around 2625 B.C.E. when the Egyptian physician Imhotep (Figure 1) described "bulging tumors of the breast". For therapy, he honestly offered only "There is none" [1].

For many subsequent centuries cancer was a not well known disease which killed only some people. It was not utill 1940 that cancer overtook many infectious diseases as an important human killer. Three decades later cancer became one of the biggest threats to global human health that takes a terrible and growing human toll. Thus current cancer pandemic is the quintessential product of modernity. The War on Cancer, the "cancer crusade" forced by the U.S. National Cancer Act of 1971 provided a massive stimulus for cancer research. The Act made big promises, promoted the U.S. National Cancer Institute (NCI) and gave NCI a token measure of independence. The NCI elaborated strategy of the war based on the existed hypothesis of cancerous somatic mutation of an alone cell and subsequent metastasis of its diseased offspring around affected human body to form secondary (metastatic or dispersed) tumors [2].

Since the 1971 act, National Cancer Institute has spent about $\$ 90$ billion on science, treatment, and prevention of cancer [3]. Now, 40 years later, the disease continues to spread throughout the globe. The efficacy of means exploited currently for cancer prevention and treatment appeared to be very low. For instance, Provenge, a most recent immune treatment for metastatic prostate cancer costs $\$ 93,000$ and extends life about 4 months [4]. Really, "There is none" for therapy of cancer. The promises of 'somatic mutation hypothesis' appeared to unpaid.

Most research and treatment questions that then vexed the cancer community remain unanswered. The initially accepted paradigm of cancer origin and pathogenesis appeared to be impotent. Nevertheless the bankrupt paradigm continues to be kept by experts predicting total U.S. spending on cancer care could rise by as much as $66 \%$ to $\$ 207$ billion by 2020 [5] without any guarantee for relevant increase of the investments' efficacy. Based on the 


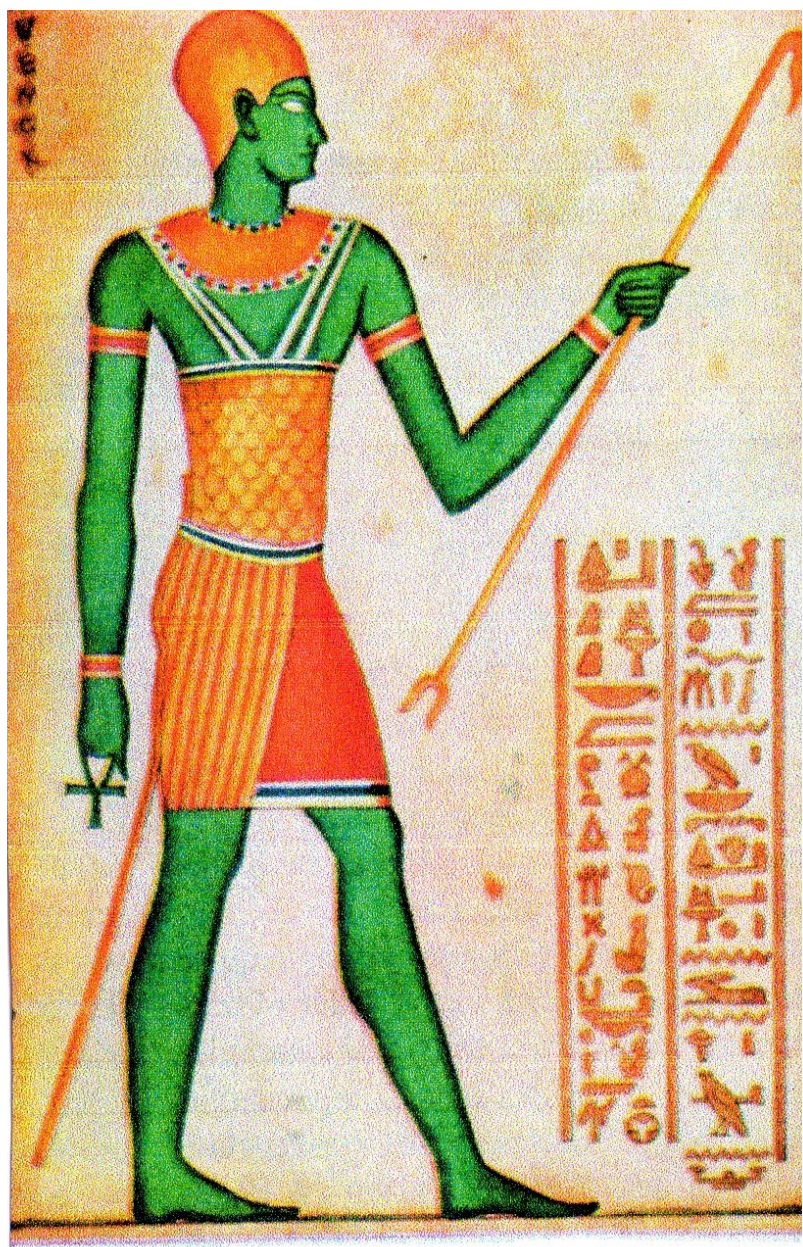

Figure 1. Imhotep. First to describe a cancer [1].

hypothesis of somatic mutations and consequent metastasis, oncology faces its limits. The search for subtle links between diet, lifestyle, or environmental factors and disease leads to an unending source of fear, but often yields little certainty. Studies on weak associations or small effects often produce contradictory results which confuse the public.

A need has emerged to develop a more enlightened paradigm that might capture the most essentials about the cancer. New insights into the origin, pathogenesis and epidemic spread of the disease are therefore sorely needed. There are many observations, experiments and theoretical discoveries to be made in this way. The present article aims to present the entire set of evidence of the bankruptcy of the 'somatic mutation hypothesis' and to promote a systematic search for such new insights which should open new view on the origin of cancer and its pathogenesis, including the dispersion of cancerous cells around the body and forces propelling this process.

The article presents the results from reconsidering and re-comprehension of various either direct or indirect data regarding cancer epidemiology, clinical manifestations, and molecular pathogenesis from the viewpoint of recent all-pathological, immunogenetic, genetic, and evolutionnary discoveries followed up to cellular, subcellular and molecular level. The main accent was on the observations of genetic predilection to cancer amongst different human populations, ethnoses, and individuals. Special attention was paid to the revealing of the signs of genetic peculiarities of different locations of cancer around diseased body. Over the comprehension of the origin of cancer the last one was considered as an entire phe- nomenon resulted from an entire process. This feature of exploited methodology was considered as condition sine qua non.

\section{RESULTS AND DISCUSSION}

\subsection{Prevalence of Cancer}

Although cancer occurs in every country in the world, there are wide ethnic variations in its mortality rates (Figure 2). The rates used are the number of cancer deaths per 100,000 population. They are ranked from the highest to the lowest. The data revealed four-fold difference between the lowest (54.4 in Thailand) and highest (235.4 in Hungary) male cancer mortality rates. The group of five most cancerous countries unites Hungary, Luxembourg, Belgium, France and Uruguay. Amongst a group of five least cancerous countries Mexico, Ecuador and Panama shares their neighborhood with Thailand and Kuwait. One can suppose in contrast to Hungary the population of Thailand could be named innately immune to cancer.

The rates of cancer incidence show far more variations [6]. The rates for all cancer sites in males revealed an over eight-fold differences that ranged from 493.8 per 100,000 in Tasmania, Australia, to a low of 59.1 in The Gambia, that shows also lowest rates for cancer of colon, rectum, pancreas, bronchus, lung, thyroid gland, myeloid leukemia, bladder, tongue, mouth and testis. One can expect the key to the origin of cancer will be found in the ecology of The Gambia innate ethnos, which provided him with more than 5-fold resistance to cancer in contrast to the USA blacks and whites. Prostate cancer, one of the most common cancers in men, is more frequent in the USA men of African origin. Large variations were observed at primary sites of skin and pancreas cancer (Figure 3).

At the same time incidence rates for all cancer sites in African Americans are $>1.5$-fold greater than rates in European Americans [7] that can be explained by 400 years old genetic closeness between the ethnoses.

The largest ratios of the highest rates to the lowest rates in worldwide cancer incidence (Table 1) among 


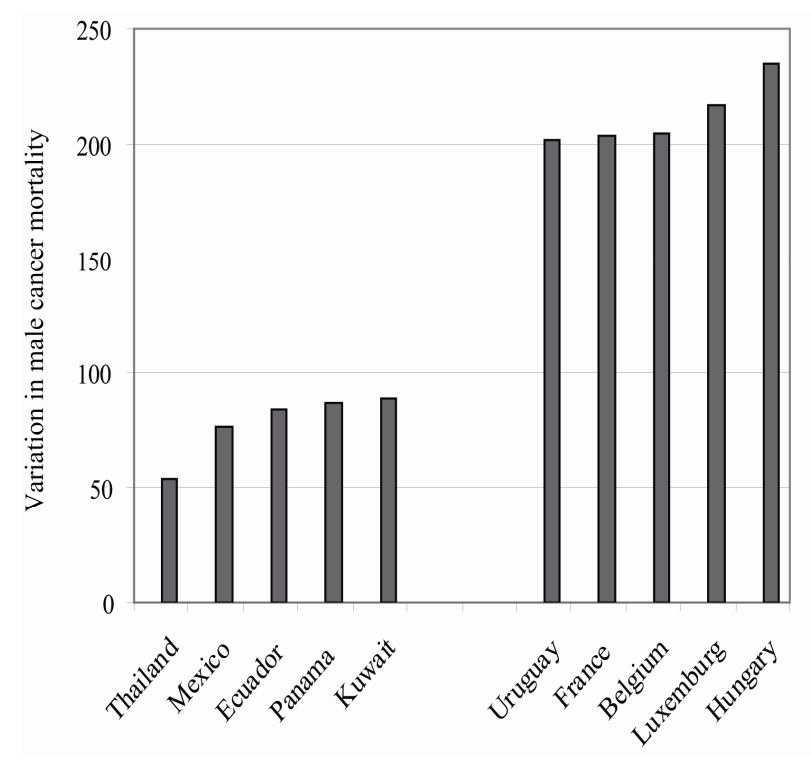

Figure 2. Variation in male cancer mortality rates among different populations according to [8].

males were for melanoma of the skin, nasopharynx, and larynx, with ratios of 289, 285, and 204, respectively. For melanoma of the skin, the area reporting the highest rate was the Australian Capital Territory with 28.9 per 100,000; the lowest rate, 0.1, was reported among Kuwaitis in Kuwait and among persons in Khon Kaen, Thailand. For nasopharynx, the highest rate was 28.5 in Hong Kong while the lowest was 0.1 for Quito, Ecuador. For larynx, the highest rate was 20.4 in Basque Country, Spain, and the lowest rate, 0.1, was for men in Qidong, China. Prostate cancer rates were highest for black men in Atlanta, Georgia (102.0) and lowest in Qidong, China $(0.8$ per 100,000$)$. The worldwide range in lung cancer incidence among men ranges from a high of 119.1 in New Zealand Maoris to 1.0 per 100,000 in The Gambia. U.S. black men in New Orleans experienced a lung cancer rate of 115.9, just lower than that for Maoris in New Zealand.

These observations (Figures 2-3 and Table 1) are seen very mysterious in the light of the orthodox postulates about the causes of cancer. This is one of the main riddles of cancer manifestations that should be decoded. At the same time, they evidenced the existence of ethnoses (and persons) with very high grades of natural i.e. genetic immunity to cancer and thus reveal very important milestones in the way to the deciphering of both the origin of cancer and the genetic components of the disease pathogenesis. A more complete understanding of cancer origin, pathogenesis and epidemic spread will come from the discovery of relevant subjects in opposite ethnic and racial groups. One of the mile stones could be the traits of ethnoses and populations which reveal opposite values of the rates of cancer prevalence. Another milestone could be revealed by the analysis and comprehension of both individual and intra-individual diversity in genetic immunity to cancer.

\subsection{Unique Features of Cancer}

Any disease displays a set of universal all-pathological features that are also character istic of other diseases. The set of universal features includes at least a dozen intrinsic signs: 1) different incidence of a disease among different races and ethnic groups, 2) increased prevalence of diseases in developed and civilized countries, 3 ) genetic predilection to the disease, 4) age differences in the disease incidence, 5) stochastic distribution of indi-

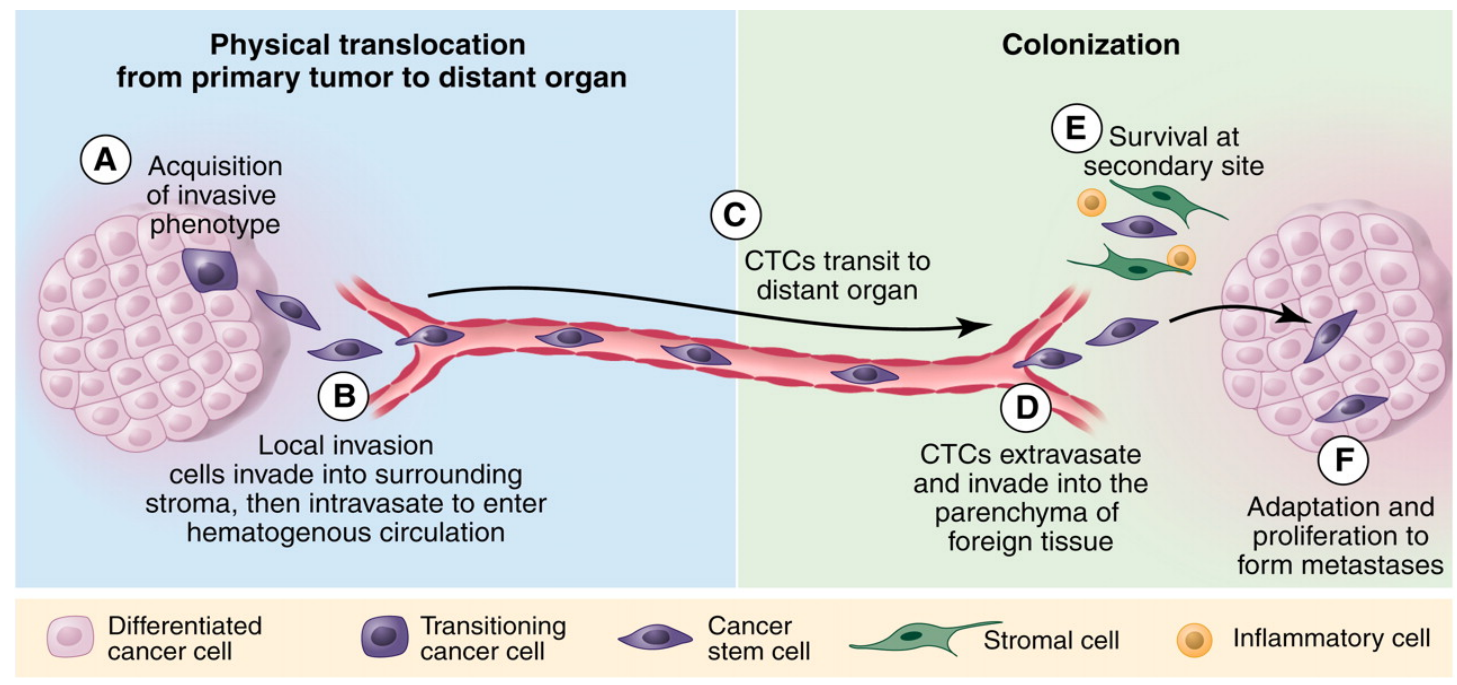

Figure 3. Electronic visualization of supposed translocation of cancerous cells from primary tumor to distant organ [9]. 
Table 1. The ratios of the highest rates to the lowest rates in worldwide cancer incidence according to [7].

\begin{tabular}{cccc}
\hline & \multicolumn{3}{c}{ The values of rates per 100,000 } \\
\cline { 2 - 3 } Cancer & Highest rates & Lowest rates & Ratio \\
\cline { 2 - 4 } $\begin{array}{c}\text { Skin } \\
\text { melanoma }\end{array}$ & 28.9 (Australia) & 0.1 (Kuwait) & 289 \\
Nasopharinx & 28.5 (Hong Kong) & 0.1 (Ecuador) & 285 \\
Larynx & 20.4 (Basque, Spain) & 0.1 (China) & 204 \\
Prostate & 102.0 (Atlanta, Ga) & 0.8 (China) & 127 \\
Lung & 119.1 (Maoris, NZ) & 1.0 (Gambia) & 119 \\
\hline
\end{tabular}

vidual cases amongst a population, 6) individual variations in constitutional (genetic) predilection to the disease, 7) the mosaicism of affections, i.e. intra-individual diversity both in the predilection of different parts of a tissue and in the quantity and sizes of affections, 8) dappled distribution of affections amongst a body, 9) molecular bases of genomic and cellular pathogenesis and $10)$ the identity of involved cells in any locations of specific affections around the body [10].

Each of these universal features expresses the allpathological phenomenon of heterozygous mosaicism created by genetic admixture arising as a result of hybridization between two genetically different organisms: one of which is constitutionally immune to the relevant ecological or physiological agent whereas its mating partner is constitutionally sensitive to it. The heterozygosity results in the coexistence of at least two activeallelomorphic genes in the offspring's genome. Both alleles function dominantly and create two allelic cell clones whose subpopulations are formed and distributed in the body before postnatal ontogenesis. The heterozygous offspring expresses both alleles equally but in different sizes and separated locations around the body. The features and functions of codominant clones may become obvious at different steps of ontogenesis [11]. This is a kind of chimerism or cellular mosaicism, the occurrence in an individual of two or more cell populations of different chromosomal constitutions, derived from different parental individuals $[12,13]$.

Genetic admixture (also called xenogamy, outbreeding, cross-fertilization, crossbreeding) refers to the reproductive union of genetically dissimilar or unrelated organisms within the same species that inevitably results in offspring heterozygosity of various kinds. The states of heterozygosity are responsible for the origin of spotted mosaic manifestations, individually different course and severity of most diseases, both infectious and non-infetious $[14,15]$. The mosaicism is revealed in genetically determined variations in the location, size and other pathological manifestation of any disease. Every human disease is extraordinarily diverse in its manifestation. Affected people may have many individual differences in the manifestations of their illnesses as well as in the grade of expression.

Each of these universal traits of pathology belongs to any form of cancer too. The shape, disposition, size and rate of cancer progression are also very different in different individuals. However, the origin and development of malignancy reveals some unique features. Firstly, in contrast to any other disease, cancer comes into sight when the division and growth of some cells in some parts of the body become uncontrolled. Secondly, the cancer cells look abnormal under the conventional light microscope. They are considered versions of cells which compose the tissue of the supposed cancer origin, however, light microscopy cannot identify the tissue and site of a malignancy origin [16]. Thirdly, cancer genetics holds some mystery traits which should be taken into account too.

\subsection{Usualness of Cancer Genetics}

Recent genetic investigations revealed a number of apparent paradoxes and alternative views of the traits of cancer genetics [17]. The undoubted genetic predilection to cancer is characteristic of both usual and unique features that can be observed at any level of the disease existence beginning from ethnic and population ones. Although it is now a well confirmed fact that genetic factors play an important role in all steps of cancer development and a person's genetic makeup has a principal influence on the fate of a patient $[18,19]$, very little is known about the special characteristics of the genome that determine the unregulated behavior of cancer cells and their distribution around the body [20]. There is known only a minority of cancer sites that arise as a result of inherited and highly penetrant cancer susceptibility genes [21]. In contrast, the genetic principle of analogous distinct distribution in both infectious and most noninfectious diseases has been deciphered [10].

Cancer rates in the Californian population of South Asians, that comprise people having origins mainly in India, Pakistan, Bangladesh and Sri Lanka, are different from those breast cancer observed in other ethnic groups inhabiting the same state. Compared to rates in native Asian Indians, rates of cancer in South Asians of California were higher for all sites of cancer locations. In contrast to Asian/Pacific Islanders of California, the South Asian population experienced more cancers of the esophagus, gall bladder, prostate, breast, ovary and uterus, as well as lymphomas, leukemias and multiple 
myelomas. Compared to the non-Hispanic White population of California, South Asians experienced more cancers of the stomach, liver and bile duct, gall bladder, cervix and multiple myelomas. Significantly increasing time trends were observed in colon and breast cancer incidence [22]. African-American women have a lower overall incidence of breast cancer than do Caucasian women, but a higher overall mortality and the differences between their breast cancer cell lines play a role in their different rates of cancer disposition around a body [23].

Recent data of cancer genome sequencing show that almost all the changes in the gene structure of cancer are heterozygous and present in nearly all the cells in the discovered tumor samples [24]. This indicates both the sameness and the unity of cancerous tissue. The malignnant phenotype is determined largely by early transforming events rather than being molded by somatic evolution during the clonal expansion of neoplastic cells [25-27]. Many other genetic findings also confronted the somatic mutation theory with a number of apparent and alternative views [17].

The genotype of cancerous cells is not identical to those of normal ones. In contrast to a well-known fact that vast diversity of normal cell phenotypes in any living body is generated by the same genome the initiation and development of cancer is influenced by the inherited cancer-promoting genotype [28,29]. Because it begins to function at the end of reproductive age, this highly pathogenic genotype has not been eliminated by natural selection.

\subsection{Specificity of Cancer Pathogenesis}

Cancer presents a group of malignant diseases characterized by abnormal reproduction of some cell clones and consequent growth of relevant tissues in different parts of afflicted bodies. At least four different kinds of such malignancies' pathogenesis were discovered among human and animals. Firstly, some forms of malignancies arise from infection with specific contagious viruses or bacteria. Secondly, there exists canine transmissible venereal tumor among dogs and analogous contagious cancer among Tasmanian devils [30], sea turtles and sea lion and so on $[31,32]$. These arose after direct physical intrusion of viable cancerous cells from one host to another either over natural sexual contacts or by laboratory manipulations of animals and, occasionally in rare circumstances, over organ transplantation. Sexually transmitted tumor of dogs has a worldwide distribution and that probably arose thousands of years ago. Most cases of this form of cancer are eventually rejected by afflicted dog, who then is conferred lifelong immunity $[31,32]$. Thirdly, there are tumors transferred from mother to fe- tus. And at last, there is cancer of predominant kind that presents one of the biggest and epidemically growing problems in the modern world whose extensive counteracting efforts appeared to be shamefully impotent. The pathogenesis of this predominant form of cancer is principally another.

Every kind of living being is constitutionally provided with a physiological system that maintains normal body structure within its genetically predetermined shape and size. Special part of this very effective system is dedicated to regulate the starting and revival of body structures and their functions on their molecular, sub-cellular, cellular, tissue and organ levels. Normally, cells grow and divide to form new cells as the body needs them. When cells grow old and die, new cells take their place. The regulation is realized on the level of cells and performed by means of hormonal molecules.

In the case of cancer this orderly process goes wrong. This mighty system of body maintenance appears of being impotent in the relation of some its initially smallest parts. That is happened because cancer is formed by of abnormal cell clone that is able to grow independently of normal physiological control. As a result its cells are forming when the body does not need them whereas some of its old cells do not die when they should. The appeared extra cells form the masses of tissue, called malignant tumors.

Two intrinsic hallmarks belong to any kind of cancer. The first and most essential hallmark is absolute resistance of cancer cells and tissues to normal physiological regulation of cell growth and tissue formation. The second hallmark is expressed in the phenomenon of absolute immunity of malignant cells and tissues to the destruction by both cell and humoral mechanisms launching by lymphatic system of responsive immunogenesis that allows cancer evade the surveillance performed by the host's immunogenic systems. Both the hallmarks perform their obligate functions in the initiation, development and subsequent progression of any kind of cancer.

The lymphatic system of responsive immunogenesis is unable to defend us from cancer's development. On the contrary, the effective cells of lymphatic system are thought to play an important role in the provocation of carcinogenesis. According to [33] and on the contrary to the hypothesis of somatic mutation the cells may induce malignant transformation of normal cells. Moreover, once cancerous cells develop, an immunoediting process occurs in which immune cells and their molecular mediators dictate the development and progression of cancer [33]. Tumor cells also develop several mechanisms to evade anti-tumor immunity by developing an immunosuppressive microenvironment. The differences in the populations of lymphatic cells infiltrating into tumor tissues 
are associated with differences in clinical outcomes [33]. The underlying molecular mechanisms of the association should be unraveled to get better understanding of the complex relationship between tumor cells and the associated lymphatic immunogenic cells.

On the other hand, the deficiencies of lymphatic immunogenic system that are present in the tumor environment enhance also the progression of the tumor in the host. Such function is thought may belong to the inhibittion of natural killer cytotoxic responses, the accumulation of myeloid suppressor cells in the tumor, deficiencies on interferon signaling, the secretion of cytokines that enhance tumor growth (i.e., IL-6, IL-10, CSF-1, TGF-b, TNF), and the expression of surface molecules (i.e., HLA-G, B7-H1, B7-H4, CD40, CD80) that have a role on immune suppression [34].

The process of origin and development of malignancy reveals some unique traits of cancer [35]. Its uniqueness is the abnormality of its cell morphology and aggressive behavior performed by uncontrollable division of cancerous cells and growth of cancerous tissue. In contrast to any other disease, cancer comes into sight when the division of cells and tissue growth become uncontrolled in some parts of the body. The disturbance is associated with the resistance of cancerous cells to relevant molecular physiological regulators of cell dividing and tissue growth. against growth inhibitory signals. This ability provides them with the capability for unlimited replication and to evade programmed cell death. This kind of specific immunity functions against ecological and physiological agents. It is known as hereditary, genetic or constitutional [36].

Hereditary immunity arises in evolution as a result of natural selection performed by life threatening molecular ecological factors of infectious, animals and plant origin. In a case of relevant ecological danger, individuals possessing a mutantly modified molecular constitution rendering them incapable of being affected with the agent appear constitutionally immune to a particular agent. They give rise to immune progeny while susceptible individuals of the same species become ill and die without reproduceing [36,37]. On repeated exposure of many generations to a given pathogen, the progeny of immune variants eventually predominate in a population; an individual protecttive variation becomes the property of a group, then of a population and, finally, of most of a species [38,39].

This kind of immunity is determined by constitutional incongruence between relevant ecological (e.g. infectious) regulator and its molecular target in the body. Analogous mechanisms perform constitutional resistance against molecular physiological regulators which are also responsible for many noninfectious diseases. The princeples of cell immunity to physiological agents are analogous to those ones in hereditary immunity to infections [10].

Hereditary immunity of cells to relevant hormonal regulators is crucial cause of many diseases. It is created by mutant modifications of either the hormone or its receptor, that forms an incongruence between the coactors, i.e. constitutional immunity against hormone influence [40-42]. The blocking effect of mutant modifications of either hormones or their receptors leads to the development of obesity [43]. Genetic immunity of cells to insulin is a major determinant of the decline of glucose tolerance. Non-insulin-dependent diabetes mellitus is characterized by pathological hyperglycemia in the presence of higher-than normal levels of plasma-insulin. A pathogenic decrease in cell sensitivity to vitamin D3 determines the familiar forms of rachitic. The immunity of cells to androgens causes the phenomenon of testicular feminization. Constitutional resistance of cells to corticosteroids determines the pathogenesis of Cushing's disease [43]. The grade of the cells immunity to thyroid hormone determines the range of relevant disturbances. This resistance is an inherited inability to respond appropriately to the $\mathrm{T} 3$ hormone linked to mutations in the thyroid hormone receptor (TR)-beta [44]. One can note that whereas the cell resistance to hormonal or infectious influences has no visible distinctions from the susceptible ones, the cancer cells look abnormal even under the conventional light microscope. They are considered versions of cells which compose the tissue of the supposed cancer origin, however, light microscopy cannot identify the tissue and site of a malignancy origin [16].

The analogous origin of cancer cells immunity against molecular physiological regulators of cells dividing and tissue growth has recently been hypothesized. The set of above data allowed explain the most unique feature of cancer, its aggressive behavior provided with uncontrollable dividing and growth of cancerous cells. It was supposed the physiological uncontrollability of cancerous cells is predetermined by their natural (genetic) immunity to the influence of relevant molecular cyto-ecological regulators of cell circle and tissue growth [35]. This supposition, together with mutual exposure, analysis and evolutionary comprehension of a set of relevant immunological data, allowed put forward a new idea about molecular pathogenesis of cancer.

\subsection{Disposition of Cancer around a Body}

A cancer may exist in an individual body either as alone alien mass (tumor) or as several discrete forms of it. Most cases of cancer are characteristic of severalty, a state of being several and discrete. In the case of discreteness, they may have more than two but not many several parts which appear visually detectable in different times and at different areas of the body. It is taken to suppose that can- 
cer can dispose in any organ or tissue of the body i.e. that any part of a body are accessible to cancer settlement. The first appeared tumor is called the 'primary' tumor. It is usually named for the part of the body or the type of cell among which it appeared. The tumors which arose later are named the secondary, metastatic or dispersed tumors. The last consist of the same type of cells and get the same name as the primary tumor.

The list of cancer names is very large. For instance, Muir et al. [45] presented the names as follow: the cancer of lip, tongue, mouth, oropharynx, nasopharynx, esophagus, stomach, colon, rectum, liver, gallbladder, pancreas, larynx, bronchus, lung, melanoma of skin, prostate, testis, penis, bladder, kidney, brain, nervous system, thyroid gland, Non-Hodgkin's Lymphoma, Hodgkin's Disease, Multiple Myeloma, Lymphoid Leukemia, Myeloid Leukemia. There are more than a hundred distinct sites where primary cancers can be disposed either alone or in the combinations with secondary ones.

At least two paradoxes can be seen in the disposition of either primary or secondary malignant tumors. Firstly, in contrast to the potential ubiquituosness of primary tumors there are both more favorite and far less favorite sites of their secondary dispositions (Table 2). The primary cancers are mainly disposed at prostate, lung \& bronchus, colon, urinary bladder, skin, kidney, rectum .pancreas, stomach. Besides, hypopharynx, bones \& joints, floor of mouth, nasopharynx, gallbladder, oropharynx, oral cavity, trachea, peritoneum and pleura are far less favorable for the disposition of primary tumors. Secondly, in contrast to the potential ubiquituosness of primary tumors there are only some most common sites where the secondary tumors are preferably dispose-the lungs, bones, liver, and brain. Other places of a body are seen far less accessible for secondary tumors. One question arise immediately-are these unfavorable places immune to the invasion of cancer? The way of living of such variation as well as its reasons have not been discussed anywhere before.

Two principal variants for explanation of the reasons of cancer's discreteness can be today. Firstly, for the last 80 years the prevailing paradigm in cancer origin and pathogenesis was exclusively based upon the "somatic mutation hypothesis" $[2,46]$, which states firstly that any case of cancer is derived from a single somatic cell that has accumulated multiple DNA mutations in genes which control cell proliferation. The mutations are resulted in unprecedentedly intensive reproduction of the transformed cell and in the formation of primary tumor inside the affected tissue. It means the disposition of any primary tumor is predestined by the location of maternal mutant cell.

The "somatic mutation hypothesis" has also supposed that some maternal cells are able to move (metastasize) outside of primary tumor mainly through the bloodstream or the lymphatic system and form several seconddary tumors in distant locations in the body mainly in the lungs, bones, liver, and brain. The dispersed disposition of cancer cells is paradigmatically considered as a result of their distant translocation (metastasis) from maternal tumor [9]. The explanation suggests that secondary tumor can be portrayed as a two-phase process: The first phase involves the physical translocation of a cancer cell to a distant organ, whereas the second encompasses the ability of the cancer cell to develop into a lesion at that distant site (Figure 4). In this way the cells should acquire invasive traits, be chipped off the mass of primary tumor, invade toward either blood or lymphatic vessel and after all exit the circulation and invade into the distant foreign tissue. Besides, cancerous cells have diameters $(20$ to $30 \mu \mathrm{m})$ that are far too large to allow them to pass through $8-\mu \mathrm{m}$ diameter bore of capillaries such as those present in the capillary beds of the lungs [9].

The "somatic mutation hypothesis" has also supposed

Table 2. Opposite rates of male cancer incidence by primary site and race*(Rates are per 100,000 persons of the 2000 U.S. standard population). *According to [47].

\begin{tabular}{|c|c|c|c|c|}
\hline & Cancer sites & All Races & White & Black \\
\hline \multicolumn{5}{|c|}{ Sites of Highest Rates } \\
\hline 1. & Prostate & 156.9 & 145.0 & 226.0 \\
\hline 2. & Lung \& Bronchus & 85.0 & 79.9 & 95.1 \\
\hline 3. & Colon & 36.9 & 36.0 & 46.1 \\
\hline 4. & Urinary Bladder & 36.0 & 37.9 & 18.3 \\
\hline 5. & Skin & 25.6 & 28.0 & 2.0 \\
\hline 6. & Non-Hodgkin & 22.6 & 23.1 & 16.0 \\
\hline 7. & L-ma & 20.8 & 20.7 & 23.1 \\
\hline 8. & Kidney & 15.8 & 15.5 & 15.9 \\
\hline 9. & Rectum & 13.2 & 13.0 & 15.7 \\
\hline 10. & Pancreas & 9.2 & 8.1 & 15.5 \\
\hline \multicolumn{5}{|c|}{ Sites of Lowest Rates } \\
\hline 1. & Hypopharynx & 1.2 & 1.1 & 2.4 \\
\hline 2. & Bones \& Joints & 1.1 & 1.1 & 0.8 \\
\hline 2. & Floor of Mouth & 0.9 & 0.9 & 1.1 \\
\hline 4. & Nasopharynx & 0.8 & 0.7 & 1.1 \\
\hline 5. & Gallbladder & 0.8 & 0.6 & 1.1 \\
\hline 6. & Oropharynx & 0.7 & 0.7 & 1.2 \\
\hline 7. & Oral cavity & 0.4 & 0.4 & 0.6 \\
\hline 8. & Trachea & 0.3 & 0.3 & 0.2 \\
\hline 9. & Peritoneum & 0.1 & 0.1 & 0.1 \\
\hline 10. & Pleura & 0.0 & 0.0 & \\
\hline
\end{tabular}




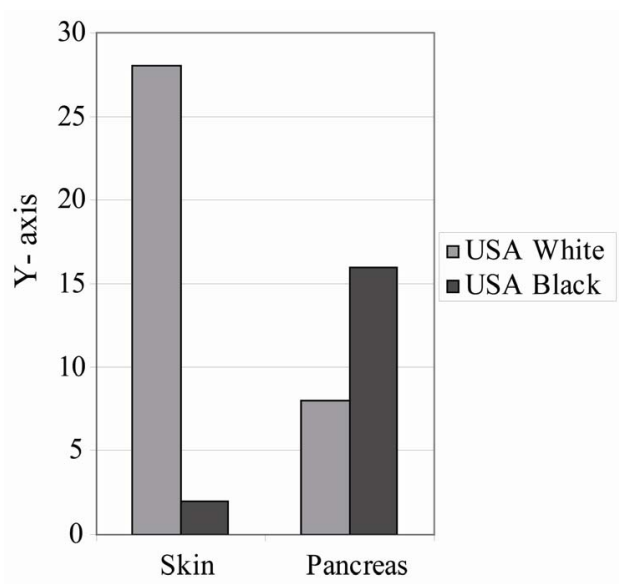

Figure 4. Opposite rates of male cancer incidence by primary site and race*(Rates are per 100,000 persons of the 2000 U.S. standard population). *According to [47].

that some maternal cells are able to move (metastasize) outside of primary tumor mainly through the bloodstream or the lymphatic system and form several seconddary tumors in distant locations in the body mainly in the lungs, bones, liver, and brain. The dispersed disposition of cancer cells is paradigmatically considered as a result of their distant translocation (metastasis) from maternal tumor [9]. The explanation suggests that secondary tumor can be portrayed as a two-phase process: The first phase involves the physical translocation of a cancer cell to a distant organ, whereas the second encompasses the ability of the cancer cell to develop into a lesion at that distant site (Figure 4). In this way the cells should acquire invasive traits, be chipped off the mass of primary tumor, invade toward either blood or lymphatic vessel and after all exit the circulation and invade into the distant foreign tissue. Besides, cancerous cells have diameters $(20 \mu \mathrm{m}$ to $30 \mu \mathrm{m})$ that are far too large to allow them to pass through $8-\mu \mathrm{m}$ diameter bore of capillaries such as those present in the capillary beds of the lungs [9].

The existence of first phase is partially confirmed: Large quantities of tumor cells can really circulate in blood and lymph channels but without overt new tumors $[48,49]$. This may mean at such cases the body does not contain the sites acceptable for realization the ability of circulated cancer cells to develop into a lesion at that distant site (second phase). Except the alone site of primary tumor the whole body is absolute immune to the inception of secondary tumors. The appearance of secondary breast cancer was reported to occur even after 20 - 25 years of disease-free period. After this time, recurrences were rare, and the mortality rate was no longer statistically significantly different from that of the general population. Patients surviving to this time without evidence of recurrence or contralateral breast cancer are probably cured [50].

Although metastasis is responsible for as much as $90 \%$ of cancer-associated mortality, yet it remains the most poorly understood component of cancer pathogenesis. This process of cancer transposition remains one of the most enigmatic aspects of the disease [9]. It remains hypothesized and mysterious [51]. The tries to envisage the hypothetical process by means of computer graphics [9] create only the illusion of truth but do not change the situation.

The somatic mutation hypothesis met recently many questionable assertions about of its main premises. The most questions have been induced by the hypothesis' supposition about the ability of maternal cancerous cells to move outside of primary tumor and cross several color lines in their ways to the lungs, bones, liver, brain and some other sites where the secondary tumors could dispose. Meanwhile the existence of the process has not been evidenced by observations. In reality we can only observe non-simultaneous appearance of several identical tumors in different parts of a diseased body. Another explanation of the reasons and propelling forces of cancer's discreteness has been proposed and developed just recently $[35,52,53]$.

\subsection{The Hypothesis of Genome Intrusion}

The opposite point of view on cancer origin, pathogenesis and pandemic spread has been presented by 'the hypothesis of genome intrusion' (HGI) based on reinterpretation and integrative re-comprehension of main pathogenetic, immunological, genetic, clinical, epidemiological and evolutionary features of the disease $[35,52,53]$. The emergence of the hypothesis has been predestined by the discovery of a set of universal all-pathological features that include at least a dozen intrinsic signs: a) different incidence of a disease among different races and ethnic groups, b) increased prevalence of diseases in developed and civilized countries, c) genetic predilection to the disease, d) age differences in the disease incidence, e) stochastic distribution of individual cases amongst a population, $\mathrm{f}$ ) individual variations in constitutional (genetic) predilection to the disease, g) the mosaicism of affections, i.e. intra-individual diversity both in the predilection of different parts of a tissue and in the quantity and sizes of affections, h) dappled distribution of affections amongst a body predestined by xenogamous genome intrusion i.e. genetic admixture, i) molecular bases of genomic and cellular pathogenesis and j) the identity of involved cells in any locations of specific affections around the body [10]. Each of these universal signs of pathology belongs to any form of cancer. 
Besides, it has been hypothesized that any cancerous cell clone is genetically alien, non-self for afflicted body $[35,53]$. It might appear in the body as a result of genetic admixture led to the intrusion of personal genome with information to control the life of foreign clone which possesses its own deviant genetic programs responsible for the dividing of cells and tissue growth. After that the clone functions according to its own program of ontogenesis including aging. From this point of view any individual cancer should be considered as a result of inappropriate foreign intrusion in a genome under consideration.

The HGI associates the emergency of cancerous cell clone with the parent's xenogamy which leads to the formation in the offspring's body of two coexisting cell clones of similar origin with opposite predisposition to both their growth regulators and the development of malignancy. The almighty lymphatic system of individual adaptive immunity does not recognize the deposited cancer cells as foreign and does not destroy them. The inserted foreign clone is not eliminated. This may mean both the emergence of cancerous clone and the dispersion of its subpopulations around the body has been performed before postnatal ontogeny.

Separated parts of the clone are stochastically dispersed around the embryo's body before postnatal ontogeny by a manner that is used to dispose other embryonic tissues and organs. After the end of their disposition the populations exist at their stable places like cell masses of smallest but different sizes. After that the clone continue to exists in the body in a form of several distantly separated populations being provided with life supporting stuffs by intruded host.

At a relevant time of a breadwinner's life (mainly after 40 years of its age) the potentially cancerous micropopulations begin to come into sight as hereditary immune against prevailing regulators of cell reproduction. The initially largest one of the cancerous micro-populations achieves detectable tumorous size far earlier in comparison to the initially smallest one. The first appeared tumor is called the 'primary' tumor. The tumors which arose later are named the 'secondary' tumors or metastases. Early diagnose and extirpation of "primary tumor" (the first appeared cancer site) may improve metastatic progression-free survival but does not exclude subsequent appearance of "secondary tumors" [54,55].

Patient age 74 years was diagnosed with stage III primary breast cancer. The volume of her primary tumor was found to be $10.3 \mathrm{~cm}^{3}$ measured through laborious reading of the whole body PET/CT scans. The tumor was resected. However, 8 years after primary diagnosis and resection, 31 bone, 3 lung, 2 lymph node, and 1 soft tissue secondary tumors were discovered (Figure 5). Volumes of all tumors were measured through laborious reading of the whole body PET/CT scans. In particular, volumes of 31 bone tumors were 1.69, 1.98, 2.01, 2.04, $2.14,2.20,2.46,3.05,3.18,3.31,3.37,3.48,3.52,3.57$, $4.22,4.34,4.73,5.04,5.08,5.25,5.45,5.64,6.36,6.55$, $7.39,9.01,9.21,11.15,12.71,13.81,22.96 \mathrm{~cm}^{3}$. Additionally, the patient had three lung tumors with the volumes $1.30,2.01$ and $7.26 \mathrm{~cm}^{3}, 2$ lymph node tumors with the volumes 2.85 and $9.66 \mathrm{~cm}^{3}$, and one soft tissue tumor with the volume $11.41 \mathrm{~cm}^{3}$ [56].

The researchers revealed also 20 and 15 secondary bone tumors in two other breast cancer patients 5.5 years and 9 months after primary resection, respectively. Besides they found the inception of the first secondary tumor occurred 29.5 years prior to the primary diagnosis, and resection of the primary tumor was followed by a 32 -fold increase in the rate of secondary tumors growth [56]. This may mean the growth of all populations of a cancer is under control performed by their own united physiological mechanism which maintains the whole structure of cancer within its genetically predetermined size. The physiological unity of cancer parts has recently been evidenced by observations on the fate of cancers partially deleted over oncologic surgical procedures. It has been shown the deletion of some tumors by partial hepatectomy initiated proliferation of other parts the cancer has been left after the surgery which resulted in a rapid growth of secondary tumors ("metastases") in the remaining liver after hepatectomy. Significant increase in tumor growth was found after 70\% hepatectomy [57].

Analogical progression of primary and secondary tu-

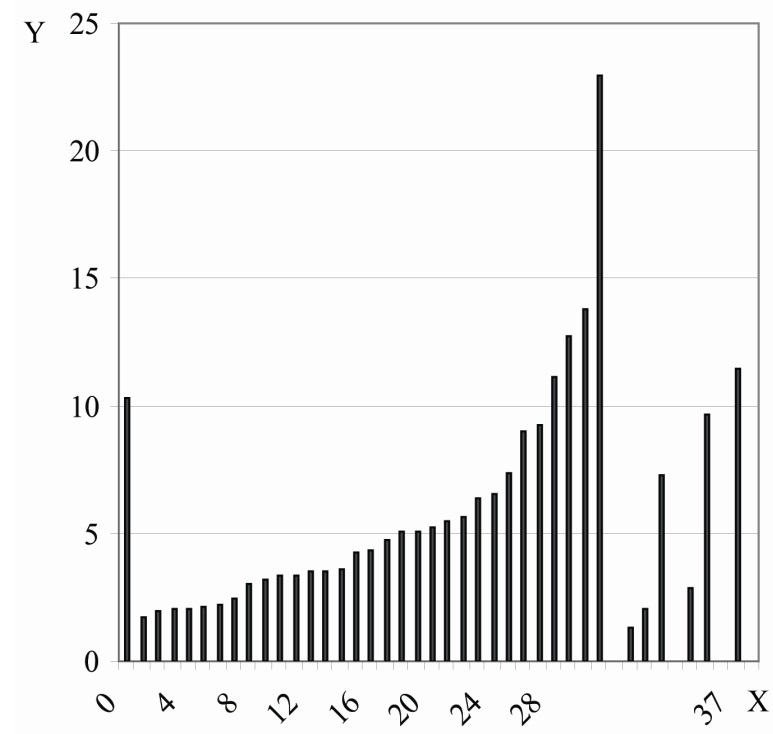

0 - primary tumor at the age 74 years. $1-37$-secondary tumors at the age 82 years (1-31-bone tumors; 32-34-lung tumors; 35 and 36-lymph nodes tumors; 37 - soft tissue tumor).

Figure 5. Volumes $\left(\mathrm{cm}^{3}\right)$ of primary tumor (before resection) and secondary tumors (8 years after extirpation of primary tumor). 
mors after foregoing resection was also noted in experimental [58-60] and clinical [61,62] studies. Partial heaptectomy impacted on the growth of tumor size in the remaining places of diseased liver. Besides the growth rate of liver's tumors was more rapid than that of the liver parenchyma. It means their growth rates are regulated by different systems. The set of dispersed parts of a cancer functions like an entire self-reliant living being settled in the affected body. That may mean cancers can produce their own growth regulators.

Cancer patients have a $20 \%$ higher risk of a new primary cancer compared with the general population [63]. As the numbers of cancer survivors and of older people increases, the occurrence of multiple primary cancers is also likely to increase [64-68]. Approximately one-third of cancer survivors aged $>60$ years were diagnosed more than once with another cancer. Possibly, these variations are associated with the phenomenon of clonal diversity in the genetic programs of the progression of senescence [69]. Such observations prompt the idea of the possible existence of a few potentially cancerous clones in the body [35] and few foreign intrusions in the genome.

\subsection{Origin of Cancer Epidemic}

Four main kinds of malignancies were discovered among human and animals. Firstly, some rare forms of malignancies arise from infection with specific contagious viruses or bacteria. For instance, infection with Rous virus can cause sarcoma among mice. Infection with human papillomavirus can induce cervical cancer among woman. Secondly, the transfer of cancer cells during sexual intercourse spreads canine transmissible venereal tumor between dogs and contagious cancer among Tasmanian devils [30], sea turtles, sea lion and so all [31,32]. Thirdly, tumors can be transferred from mother to fetus, by laboratory manipulations of animals or, occasionally, by organ transplantation. And at last, the cancer of predominant kind spreads among humans by means of genome intrusions over xenogamous self-reproduction. This kind of cancer presents one of the biggest and epidemically growing problems in the world health $[35,53]$.

The application of above scheme of cancer pathogenesis to the epidemiology of predominant human cancer can help to explain the leading cancer propelling causes of current epidemic progression. According to the above performed epidemiological and pathogenetic analysis, the carcinogenic functions of genome mutations possess important roles in the pathogenesis of relevant forms of the disease. Regretfully, none of such mutations by themselves are able to explain the pandemic spread of cancer. None carcinogenic mutations could be widely disseminated in the humankind because their rarity, randomness, and to the counteraction of na- tural selection. Thus, the undoubted existence of mutative carcinogenesis cannot be used for the explanation of the moving forces of current pandemic spread of malignancy.

In contrast, the distributive potencies of xenogamous carcinogenesis are fare more productive. The currently observed increasing incidence of most diseases [63] depends on the intensity of the genetic admixture within ethnically mixed populations [10]. Causative function of xenogamy in the origin, individual manifestations and course of malignant diseases is also evidenced by a plethora of epidemiological and clinical observations and investigations [35]. African-Americans are more likely to die from cancer then any other racial or ethnic population. In contrast, Hispanics, Asian Americans and Pacific Islanders have lower incidence rates than Whites for the most common cancers [63]. The frequency of any site of cancer varies around the world. Colorectal site of malignancy is common in the Western world and is rare in Asia and Africa [63].

Although only one cancerous clone usually exists in an affected body, the presence of a number of cancerous clones has also been documented. In a population of a developed country with high survival rates, multiple cancers often comprise two or more primary cancers occurring in an individual that originate in a primary site or tissue and are neither an extension, nor a recurrence or metastasis [68].

Xenogamous forces of cancer distribution could begin to function among humankind at the earliest steps of its evolution. Any evolutionary process is performed by two main propelling forces: mutative diversification in diversity can be enriched by interbreeding with related populations and species. For instance, the hybridization and exchange of genes between mutual ancestors of chimps and humans may have occurred over period of just a few million years. They may have interbred for a long time after their two lineages began to split apart evolutionarily [70]. Considerable admixture between genomes of Neandertals and early modern Europeans happened near 30,000 years ago [71].

The exodus out of North East Africa and subsequent dispersion around the world over the last 60,000 years has resulted in a wide biological diversification of the species and a strong self-segregation of its tribes from each other. Some tribes moved back to tropical South Africa, the homeland of their predecessors. Other groups migrated in the Euro-Asian or South-Asian ways. Their further evolution was performed by the forces, which propelled biological and social diversification of the species over its dispersion around the world. Inhabiting ecologically disparate geographical areas, migrants continued to evolve independently into five anatomically 
different races and a multiplicity of segregated ethnic groups [72]. These new ways of life did not favor a xenogamous epidemic spread of cancer, except when segregation was broken forcedly, for instance, by aggressive tribes. In contrast, the influence of xenogamy on the distribution of cancer among the members of separated ethnic groups was restricted.

Today, the situation is becoming the opposite. Thanks to growing industrialization, urbanization, globalization, and migration, most urban populations became ethnically mixed. The genomes of modern urbanized humans become the mosaics composed of genetic segments inherited from an extensive row of ancestors has been ethnically segregated before. The spread of cancer became pandemic, intensified by the growing expansion of xenogamy, the reproductive intercourses between ethnoses, which proceeded at different environmental conditions for previous evolution. The currently observed increasing incidence of cancer, as well as many other diseases, depends on the intensity of the population's genetic admixture promoted within ethnically mixed populations. This kind of pathology is now more characteristic of any mixed population. The current pandemic spread of cancer is intensified by the growing expansion of xenogamy.

\section{CONCLUSIONS}

The above-presented results of reconsideration of the actual data regarding cancer from the viewpoint of recent all-pathological, epidemiological, immunological, clinical, genetic, and evolutionary discoveries allowed a new integrative paradigm-the hypothesis of genome intrusion-about the origin and pandemic spread of the disease to be formed. Main postulates of the hypothesis of genome intrusion can be presented as follow:

1) The existence of cancer diseases is predetermined by genome mutations have created inter-ethnic differences in molecular constitution of inherent physiological systems responsible for regulation of cell dividing and tissue growth.

2) The development of individual cancer disease is initiated by the appearance in afflicted body of cell clone (or clones) inherently immune to normal physiological regulation of cell growth and tissue formation. The cells of such inherently immune clones are able to grow independently of physiological control of normal cell replication. This clone is foreign (alien, non-self) for afflicted body with many of its traits.

3) Such inherently immune clones appear in a body as a result of xenogamy (genetic admixture) led to both the intrusion of offspring's personal genome with heterozygous information and to the formation in the offspring's body of coexisting cell clones with opposite relation to the regulators of their growth and with their own deviant genetic programs of ontogenesis.

4) The emergence of cancerous clone and the discrete dispersion of its micro-populations around the body are performed before postnatal ontogeny in the manner used to dispose other embryonic tissues and organs. Thus the lymphatic system of individual adaptive immunity does not recognize the deposited cancer cells as foreign and does not destroy them. After the end of their disposition the subpopulations exist at their stable places like cell masses of smallest but different sizes being provided with life supporting stuffs by intruded host.

5) At a relevant time of a breadwinner's life (mainly after 40 years of its age), the clone gets specific impulse to awake probably either from its specific program of ontogenesis or from relevant physiological or ecological carcinogens. Its subpopulations begin to replicate uncontrollably and comes into sight in the form of detectable extra cells masses of cancerous tissue, the malignant tumors. The initially largest one of subpopulations achieves detectable tumorous size far earlier in comparison to the initially smallest one. The first appeared cell mass is called the "primary" tumor.

6) The growth of all subpopulations of a cancerous clone is under control performed by their own united physiological mechanism which maintains the whole structure of cancer within its genetically predetermined size. The destruction of one or more tumors gives boost to growth of other sub-populations of the clone.

7) None carcinogenic mutations could be widely disseminated in the humankind because their rarity, randomness, and to the counteraction of natural selection. The currently observed increasing incidence of the disease depends on the intensity of xenogamous genetic admixture within ethnically mixed populations.

The study has been performed by exposure and analysis of various epidemiological, clinical, immunological, genetic, and experimental data concerning principal characteristics common for both cancer and other kinds of diseases, especially of hormonal ones. This approach allowed expose and highlight new ways toward the discovery of molecular level of immunogenic and genetic factors involved in the appearance, evolution, spreading, and maintenance of cancer.

At least four decisive factors are involved in the creation of malignancy: 1) Natural selection for hereditary immunity against life-threatening molecular ecological agents; 2) Ethnic diversification of humankind; 3) Inter-ethnic crossbreeding; and 4) Globalization of humankind. Points 1 to 3 predestined the origin of the disease, whereas point 4 formed prerequisites and propelling forces for its pandemic spread.

The revealed set of evidences allows for the demand 
that the origin of cancer is based on xenogamous intrusion into individual genomes of relative but structurally foreign components able to control the development of cell clones constitutionally immune to physiological regulators prevalent at the intruded body. Like any other disease, cancer is characteristic of diversity in the course, manifestations, and severity of specific affections, as well as their sizes and stochastic focal disposition around the body. Individual differences in the manifestations and severity of discussed disease are associated with the phenomenon of stochastic focal distribution of cancerous zones around a body. The differences are of genetic origin. This phenomenon is analogous to those characteristics of any other kind of pathology, being explained by the hybridization of persons possessing different grades of genetic predisposition to relevant pathogens.

The methodological approach used in the performed study allowed present the first genetic explanation for the epidemic increase in cancer incidence. Like any other hormonal disturbance, cancer arises as a result of constitutional incongruence between relevant hormonal regulators and their receptors. The cancerous molecular make-up could arise and spread among the worldwide population because of xenogamy-crossbreeding among mutually distinct parents. From this point of view, the life-threatening disease could be considered as a reckoning, both for the life-saving evolution of beneficial genetic immunity to relevant ecological agents and for the production of offspring unlike their parents.

The integrative view of the origin of cancer and its spread around the world supplies a framework for understanding the genetic nature of cancer pandemic and its rising incidence in the current worldwide population. The new paradigm allows a new explanation of the origin of cancer and its pandemics as well as to launch a more complete discovery of inherited either susceptibility or immunity to cancer, for instance, by the deciphering of phenetic functions of the genome's region of $8 \mathrm{q} 24$ responsible for prostate cancer in Americans men of African origin as well as molecular make-up of the immunity of cancerous cells to relevant regulatory systems, with potential applications for prevention and treatment. It also forces to reconsider the perspective of future investigations and to reassess the principles for cancer prevention and healing. The design of families with future cancer-free genealogy and the restriction of xenogamy should get their place in the discussion about perspective approaches and investigations for cancer prevention. The impact of proposed alternative hypotheses on the future outcome of cancer therapy is expected first of all in the development of methods and means for the suppression of genetic unresponsiveness (hereditary immunity) of cancer cells to physiological regulation of cell dividing.

\section{REFERENCES}

[1] Pederson, P. (2011) On cancer and people. Science, 332, 423. doi: $10.1126 /$ science. 1206460

[2] Bauer, K.H. (1928) Mutationstheorie der geschswulstentstehung. Springer, Berlin.

[3] Marshall, E. (2011) Cancer research and the $\$ 90$ billion metaphor. Science, 331, 1540-1541. doi:10.1126/science.331.6024.1540-a

[4] Anonymous (2011) 40 years of the war on cancer. Science, 331, 1540-1544. doi:10.1126/science.331.6024.1540-b

[5] Malakoff, D. (2011) Can treatment costs be tamed? Science, 331, 1545-1547. doi:10.1126/science.331.6024.1545

[6] National Cancer Institute (1992) International range of cancer incidence. World Health Organization.

[7] Haiman, C.A., Chen, G.K., Blot, W.J., Strom, S.S., Berndt, S.I., et al. (2011) Characterizing genetic risk at known prostate cancer susceptibility loci in African Americans. The Public Library of Science, Genet, 7.

[8] National Cancer Institute (1992) Cancer death rates among 50 countries. American Cancer Society.

[9] Chaffer, C.L. and Weinberg, R.A. (2011) A perspective on cancer cell metastasis. Science, 331, 1559-1564. doi:10.1126/science. 1203543

[10] Rumyantsev, S.N. (2008) Hereditary immunity: Fundamental principles and exploitation in life study and health care. Nova Biomedical Books, New York.

[11] Rumyantsev, S.N., Shabalow, N.P., Pyasetskaya, M.F., Rogacheva, N.M. and Bardakova, L.I. (2000) Species, population and age diversity in cell resistance to adhesion of Neisseria meningitidis serogroups A, B and C. Microbes and Infection, 2, 447-453. doi:10.1016/S1286-4579(00)00320-8

[12] Bonnicksen, A.L. (2009) Chimeras, hybrids, and interspecies research: Politics and policymaking. Georgetown University Press, Washington, D. C.

[13] McLaren, A. (1976) Mammalian chimaeras. Cambridge University Press, Cambridge.

[14] Rumyantsev, S.N. (2006) The origin of individual differrences in the course and severity of diseases. The Scientific World Journal, 6, 1674-1704. doi:10.1100/tsw. 2006.278

[15] Rumyantsev, S.N. and Gerasimov, V.K. (2007) The origin and functions of biodiversity in infectious and noninfectious diseases. In: Schwartz, J., Ed., Focus on Biodiversity Research, Nova Science Publishers, Hauppauge, 199-300.

[16] Briasoulis, E. and Pavlidis, N. (1997) Cancer of unknown primary origin. The Oncologist, 2, 142-152.

[17] Soto, A. M. and Sonnenschein, C. (2004) The somatic mutation theory of cancer: Growing problems with the paradigm? Bioessays, 26, 1097-1107. doi:10.1002/bies.20087

[18] Hemminki, K., Li, X. and Czene, K. (2004) Familial risk of cancer: data for clinical counseling and cancer genetics. International Journal of Cancer, 108, 109-114. $\underline{\text { doi: } 10.1002 / \text { ijc. } 11478}$ 
[19] Ponz De Leon, M. (1994) Familial and hereditary tumors. Springer, Berlin.

[20] Heike, A. Helga, R. Simone, F. (2008) Hereditary tumor: From genes to clinical consequences. Wiley-VCH Verlag $\mathrm{GmbH}$, Weinheim.

[21] Hodgson, H. (2008) Mechanisms of inherited cancer susceptibility. Journal Zhejiang University Science B, 9, 1-4. doi:10.1631/jzus.B073001

[22] Jain, R.V., Mills, P.K. and Parikh-Patel, A. (2005) Cancer incidence in the south Asian population of California, 1988-2000. Journal of Carcinogenesis, 4, 21.

[23] Yancy, H.F., Mason, J.A., Peters, S, Thompson, C.E., Littleton, G.K., Jett, M. and Day, A.A. (2007) Metastatic pro- gression and gene expression between breast cancer cell lines from African American and Caucasian women. Journal of Carcinogenesis, 6, 8 .

[24] Mardis, E.R., Ding, L., Dooling, D.J., et al. (2009) Recurring mutations found by sequencing an acute myeloid leukemia genome. New England Journal of Medicine, 361, 1058-1066.doi:10.1056/NEJMoa0903840

[25] Bernards, R. and Weinberg, R. A. (2002) Progression puzzle. Nature, 418, 823. doi:10.1038/418823a

[26] Liotta, L.A. and Kohn, E. C. (2003) Cancer's deadly signature. Nature Genetics, 33, 10-11. doi:10.1038/ng0103-10

[27] Weigelt, B., van't Veer, L. J. (2004) Hard-wired genotype in metastatic breast cancer. Cell Cycle, 3, 756-757. doi:10.4161/cc.3.6.923

[28] Podsypanina, K., Li, Y. and Varmus, H.E. (2004) Evolution of somatic mutations in mammary tumors in transgenic mice is influenced by the inherited genotype. BMC Medicine, 15, 24. doi:10.1186/1741-7015-2-24

[29] Podsypanina, K., Du, Y.C., Jechlinger, M., Beverly, L.J., Hambardzumyan, D. and Varmus, H. (2008) Seeding and propagation of untransformed mouse mammary cells in the lung. Science, 321, 1841-1844. doi:10.1126/science.1161621

[30] Murchison, E.P. (2008) Clonally transmissible cancers in dogs and Tasmanian devils. Oncogene, 27, S19-S30. doi:10.1038/onc. 2009.350

[31] McAloose, D. and Newton, A.L. (2009) Wildlife cancer: A conservation perspective. Nature Reviews Cancer, 9, 517-526. doi:10.1038/nrc2665

[32] Welsh, J.S. (2011) Contagious cancer. Oncologist, 16, 1-4. doi:10.1634/theoncologist.2010-0301

[33] Thompson, M.S. and Mok, S.C. (2009) Immunopathogenesis of ovarian cancer. Minerva Medica, 100, 357-370.

[34] Torres, M.P., Ponnusamy, M.P., Lakshmanan, I. and Batra, S.K. (2009) Immunopathogenesis of ovarian cancer. Minerva Medica, 100, 385-400.

[35] Rumyantsev, S.N. (2010) Hypothesis: Towards the origin of cancer epidemics and pathogenesis. Journal of Carcinogenesis, 9, 1-7. doi:10.4103/1477-3163.61265

[36] Boyd, W.C. (1966) Fundamentals of immunology. Interscience Publishers, New York.

[37] Haldane, J.B.S. (1949) Disease and evolution. La Riserca Science, 19S, 68-76.

[38] Rumyantsev, S.N. (1992) Observations on constitutional resistance to infection. Immunology Today, 13, 184-187. doi:10.1016/0167-5699(92)90124-P

[39] Rumyantsev, S.N. (1998) Constitutional and non-specific immunity to infection. Revue Scientifique et Technique del Office International des Epizooties, 17, 26-42.

[40] Friedman, J.M. (2004) Modern science versus the stigma of obesity. Nature Medicine, 10, 563-569. doi:10.1038/nm0604-563

[41] Montague, C.T., Farooqi, I.S., Whitehead, J.P., Soos, M.A., Rau, H., Wareham, N.J., Sewter, C.P., Digby, J.E., Mohammed, S.N., Hurst, J.A., Cheetham, C.H. and O'Rahilly, S. (1997) Congenital leptin deficiency is associated with severe early-onset obesity in humans. Nature, 387, 903-908. doi:10.1038/43185

[42] Stunkard, A.J., Harris, J.R., Pedersen, N.L. and McClean, G.E. (1990) The body-mass index of twins who have been reared apart. New England Journal of Medicine, 322, 1483-1487. doi:10.1056/NEJM199005243222102

[43] Rumyantsev, S.N. (2006) Obesity: A reckoning both for genetic immunity to infection and xenogamy. Medical Hypothesis, 66, 535-540.doi:10.1016/j.mehy.2005.09.014

[44] Wan, W., Farboud, B. and Privalsky, M.L. (2005) Pituitary resistance to thyroid hormone (RTH)-syndrome is associated with T3 receptor mutants that selectively impair $\{$ beta $\} 2$ isoform function. Molecular Endocrinology, 19, 1529-1542. doi:10.1210/me.2005-0014

[45] Muir, C.S., Whelan, S.L., Gao, Y.T., Ferlay, J. and Powell, J. (1992) Cancer incidence in five continents. World Health Organization, Lyon France.

[46] Lockhart-Mummery, J.P. (1932) A paper on the origin of tumors. British Medical Journal, 1, 785-787. doi:10.1136/bmj.1.3721.785

[47] (2007) 1999-2007 cancer incidence and mortality data. National Program of Cancer Registries. Betesda, Maryland, USA CDC.

[48] Jiao, X. and Krasna, M.J. (2002) Clinical significance of micrometastasis in lung and esophageal cancer: A new paradigm in thoracic oncology. The Annals of Thoracic Surgery, 74, 278-284.

doi:10.1016/S0003-4975(01)03376-8

[49] Pantel, K. and Otte, M. (2001) Occult micrometastasis: Enrichment, identification and characterization of single disseminated tumour cells. Seminars in Cancer Biology, 11, 327-337. doi:10.1006/scbi.2001.0388

[50] Karrison, T.G., Ferguson, D.J. and Meier, P. (1999) Dormancy of mammary carcinoma after mastectomy. Journal of the National Cancer Institute, 91, 80-85. doi:10.1093/jnci/91.1.80

[51] Sahai, E. (2007) Illuminating the metastatic process. Nature Reviews Cancer, 7, 737-749. doi:10.1038/nrc2229

[52] Rumyantsev, S.N. (2009) The discredit of cancer metastasis. Science Advisory Board.

http://www.scienceboard.net/community/perspectives. 22 7.html.

[53] Rumyantsev, S.N. (2009) The uniqueness and ordinariness of cancer origin and pathogenesis: New epide- miological, clinical and preventive perspectives. Journal of Clinical Medicina Research, 1, pp. 32-36.

[54] Pockaj, B.A., Wasif, N., Dueck, A.C., Wigle, D.A., Boughey, J.C., Degnim, A.C., Gray, R.J., McLaughlin, S.A., Northfelt, D.W., Sticca, R.P., Jakub, J.W. and Perez, E.A. (2010) Metastasectomy and surgical resection of the primary tumor in patients with stage IV breast cancer: Time for a second look? Annals Surgical Oncology, 17, 2419-2426. doi:10.1245/s10434-010-1016-1

[55] Giuliano, A.E., Hunt, K.K., Ballman, K.V., Beitsch, P.D., 
Whitworth, P.W., Blumencranz, P.W., Leitch, A.M., Saha, S., McCall, L.M. and Morrow, M. (2011) Axillary dissection vs no axillary dissection in women with invasive breast cancer and sentinel node metastasis: A randomized clinical trial. The Journal of the American Medical Association, 305, 569-575. doi:10.1001/jama.2011.90

[56] Hanin, L. and Korosteleva, O. (2010) Does extirpation of the primary breast tumor give boost to growth of metastases? Evidence revealed by mathematical modeling. Mathematical Biosciences, 223, 133-141. doi:10.1016/j.mbs.2009.11.006

[57] Sorin, V., Mizrahi, A., Ohana, P., Ayesh, S., Birman, T., Hochberg, A. and Czerniak, A. (2009) Partial heap- tectomy in rats results in significant growth of liver metastases by increased expression of $\mathrm{H} 19$ gene. Cancer Therapy, 7, 240-244.

[58] De Jong, K.P., Lont, H.E., Bijma, A.M., Brouwers, M.A., de Vries, E.G., van Veen, M.L., Marquet, R.L., Slooff, M.J. and Terpstra, O.T. (1995) The effect of partial heaptectomy on tumor growth in rats: In vivo and in vitro studies. Hepatology, 22, 1263-1272. doi:10.1002/hep.1840220436

[59] Garcia-Alonso, I., Palomares, T., Alonso, A., Portugal, V., Castro, B., Carames, J. and Mendez, J. (2003) Effect of hepatic resection on development of liver metastasis. Revista Española de Enfermedades Digestivas, 95, 765-767.

[60] Ikeda, Y., Matsumata, T., Takenaka, K., Sasaki, O., Soejima, K., Sugimachi, K. (1995) Preliminary report of tumor metastasis during liver regeneration after hepatic resection in rats. European Journal of Surgical Oncology, 21, 188-190. doi:10.1016/S0748-7983(95)90468-9

[61] Elias, D., De Baere, T., Roche, A., Ducreux, M., Leclere, J. and Lasser, P. (1999) During liver regeneration following right portal embolization growth rate of liver metastases is more rapid than that of the liver parenchyma. British Journal of Surgery, 86, 784-788. doi:10.1046/j.1365-2168.1999.01154.x

[62] Von Schweinitz, D., Fuchs, J., Gluer, S. and Pietsch, T. (1998) The occurrence of liver growth factor in heap-toblastoma. European Journal of Pediatric Surgery, 8, 133-136. doi:10.1055/s-2008-1071139

[63] Cancer facts \& figures. (2009) American Cancer Society, Atlanta.
[64] Levi, F., Randimbison, L., Te, V.C., Conconi, M.M. and La Vecchia, C. (2008) Risk of prostate, breast and colorectal cancer after skin cancer diagnosis. International Journal of Cancer, 123, 2899-3001. doi:10.1002/ijc. 23816

[65] Milan, T., Pukkala, E., Verkasalo, P.K., Kaprio, J., Jansen, C.T., Koskenvuo, M. and Teppo, L. (2000) Subsequent primary cancers after basal-cell carcinoma: a nationwide study in Finland from 1953 to 1995. International Journal of Cancer, 87, 283-288. doi:10.1002/1097-0215(20000715)87:2<283::AID-IJC21 $>3.0 . \mathrm{CO} ; 2-\mathrm{I}$

[66] Nugent, Z., Demers, A.A., Wiseman, M.C., Mihalcioiu, C. and Kliewer, E.V. (2005) Risk of second primary cancer and death following a diagnosis of nonmelanoma skin cancer. Cancer Epidemiol Biomarkers and Prevention, 14, 2584-2590. doi:10.1158/1055-9965.EPI-05-0379

[67] Soerjomataram, I., Louwman, W.J., Lemmens, V.E., Coebergh, J.W. and de Vries, E. (2008) Are patients with skin cancer at lower risk of developing colorectal or breast cancer? American Journal of Epidemiology, 167, 14211429. doi:10.1093/aje/kwn077

[68] Soerjomataram, I. and Coebergh, J.W. (2009) Epidemiology of multiple primary cancers. Methods in Molecular Biology, 471, 85-105. doi:10.1007/978-1-59745-416-2 5

[69] Rumyantsev, S.N. (2003) The intra-individual diversity in senescence. Biogerontology, 4, 71-178. doi:10.1023/A:1024137418419

[70] Patterson, N., Richter, D.J., Gnerre, S., et al. (2006) Genetic evidence for complex speciation of humans and chimpanzees. Nature, 441, 1103-1108. doi:10.1038/nature04789

[71] Soficaru, A., Dobos, A. and Trinkaus, E. (2006) Early modern humans from the Pestera Muierii, Baia de Fier, Romania. Proceedings of the National Academy of Sciences of the USA, 103, 17196-17201. doi: $10.1073 /$ pnas.0608443103

[72] Rumyantsev, S.N. (2010) Bioecology of pleistocenic spurt in anthropogenesis. International Journal of Integrative Biology, 10, 14-21. 